\title{
37
}

\section{Effects of Superfluidity on Spheroidal Oscillations of Neutron Stars}

\author{
UMIN LEE, T.J.B. COLLINS AND H.M. VAN HORN \\ Department of Physics and Astronomy and C. E. Kenneth Mees Observatory, \\ University of Rochester, Rochester, NY 14627-0011, C.S.A.

\section{R.I. EPSTEIN} \\ Los Alamos National Laboratory, MS D496, Los Alamos, NM 87545, C.S.A.
}

\begin{abstract}
In the limit of short wavelengths, it has been shown that superfluidity significantly affects wave propagation in neutron stars. Here we abandon the short-wavelength restriction and extend these calculations to global oscillation modes. In the present analysis, the solid crust of the neutron star is divided into an outer crust and an inner crust, and a superfluid of neutrons coexists with the solid lattice in the inner crust. We have computed several low-order global spheroidal modes for $l=2$ both with and without superfluidity. We find that superfluidity in the inner crust affects the frequency spectra of acoustic $(p-)$ modes, shear ( $\left.s^{-}\right)$modes, and interfacial $(i-)$ modes, although the surface gravity $(g-)$ modes are not affected at all.
\end{abstract}

\subsection{Introduction}

Most previous calculations of the non-radial oscillations of neutron stars have completely neglected the effects of superfluidity (cf McDermott, Van Horn, and Hansen 1988 and references therein). Epstein (1988) has previously considered superfluid effects, but only in the short-wavelength limit, where the length scales for variations in equilibrium quantities are all assumed to be much longer than the typical wavelength of an oscillation. In general, global oscillations may be either spheroidal or toroidal in character. Van Horn and Epsetein (1990) extended Epstein's short-wavelength results to include the global toroidal oscillation modes of neutron stars. More recently, Mendell (1991) and his colleagues have also considered the effects of superfluidity, but they employed simple models for neutron stars, and their analysis did not reflect the variety of oscillation modes of realistic neutron stars. Although our anlalysis does not consider superfluidity in the core, we 
use a more realistic neutron star model to investigate the effects of superfluidity in the inner crust on various oscillation modes of neutron stars. The goal of the present calculations is to extend these previous analyses to the global spheroidal oscillation spectra of neutron stars.

In the two-fluid model of superfluidity, matter is represented as a mixture consisting of a superfluid component coexisting with a normal fluid. For all but the youngest neutron stars the temperature $k_{B} T \leq 1 \mathrm{keV}$ (where $k_{B}$ is the Boltzmann constant) is much less than the energy gap $\delta \sim 1-2 \mathrm{MeV}$ predicted for the superfluid (Epstein 1988 and references therein). Thus the normal fraction is vanishingly small, and we neglect it completely in the following pulsational analysis.

An object traveling through a superfluid more slowly than a critical velocity encounters no resistance. Since the critical velocity of the superfluid in a neutron star is on the order of the speed of light, one would initially expect no coupling between the solid lattice in the inner crust and the neutron superfluid. However, the neutron superfluid also penetrates the nuclei, where it exists at a much higher density. Thus, movement of the nuclei requires propagation of regions of increased density through the fluid. This results in an effective drag force on nuclei moving through the superfluid, coupling the two (Epstein 1988).

As in Epstein (1988) and Van Horn and Epstein (1990), the interaction between the lattice and the fluid is modeled by a viscous coupling between the two media. The Lagrangian perturbations of the lattice and superfluid stress tensors are, respectively,

$$
\delta \sigma_{i j}^{N}=K^{N} u_{l l} \delta_{i j}+2 \mu\left(u_{i j}-\frac{1}{3} u_{l l} \delta_{i j}\right)+C w_{l l} \delta_{i j}
$$

and

$$
\delta \sigma_{i j}^{S}=K^{S} w_{l l} \delta_{i j}+C^{\prime} u_{l l} \delta_{i j}
$$

where the quantities $u_{i j}$ and $w_{i j}$ are the strain tensors of the lattice and fluid $\left(u_{11} \equiv u_{11}+u_{22}+u_{33}\right), \mu$ is the shear modulus of the solid lattice, the $K$ s are bulk moduli, and $C$ is the coupling coefficient between the two media. The superscripts $S$ and $N$ denote the quantities associated with the superfluid and normal matter, respectively. The stress tensors defined above lead to coupled momentum equations, which govern the oscillations of the two media.

In this study, nonradial oscillations of neutron stars are investigated within the framework of the Newtonian theory of stellar oscillations. Oscillations are assumed to be adiabatic, and no thermal effects associated with 

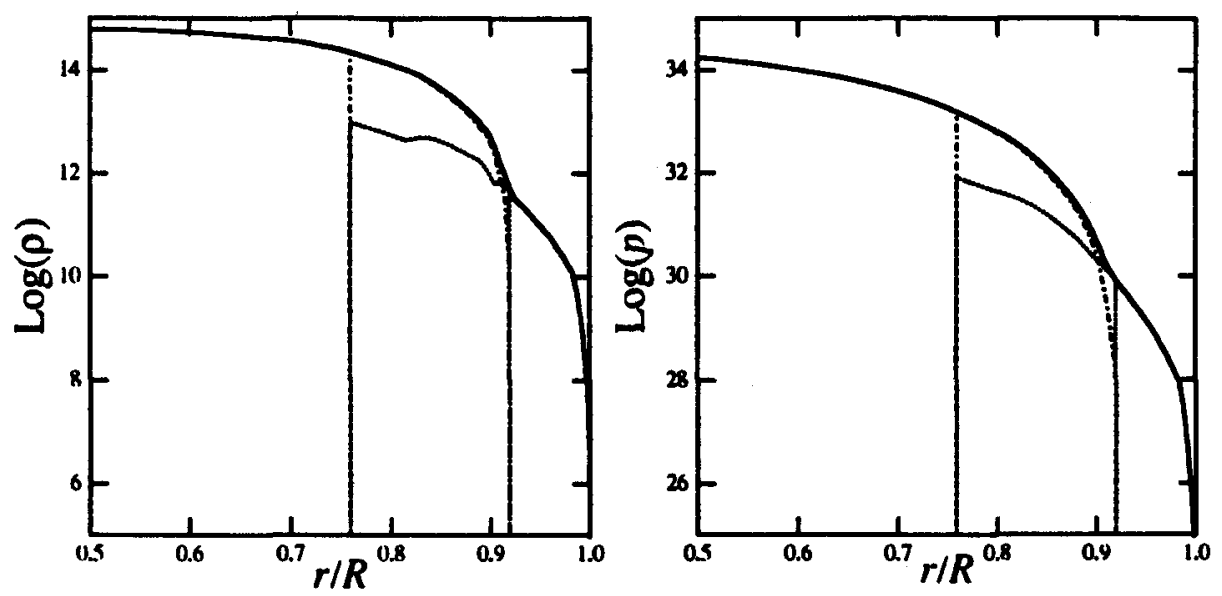

Fig. 37.1 The densities and pressures as functions of the fractional radius. The solid lines denote the total density $\rho\left(=\rho^{N}+\rho^{S}\right)$ and pressure $p=$ $\left(p^{N}+p^{S}\right)$. The dash-dotted lines denote $\rho^{S}$ and $p^{S}$ in the inner crust, while the dot.ted lines correspond to $\rho^{N}$ and $\mu^{N}$ in the inner crust.

oscillations are included. We neglect the effects of magnetic fields and rotation on the oscillations. We employ the Cowling approximation and ignore the Eulerian perturbation of the gravitational potential.

\subsection{Numerical Results}

The physical parameters of the unperturbed neutron star model used in the present calculations are $M_{*}=0.503 M_{\odot}, R_{*}=9.839 \mathrm{~km}, T_{c}=1.03 \times 10^{7} \mathrm{~K}$, and $\rho_{c}=9.44 \times 10^{14} \mathrm{~g} \mathrm{~cm}^{-3}$. We have computed the shear modulus for this model from the expression $\mu=0.2945 Z^{2} e^{2} n_{N}^{4 / 3}$ (Baym and Pines 1971), using the the relation between the number density $n_{N}$ of nuclei with charge $Z$ and the mass density $\rho$ as given by Negele and Vautherin (1973). Above the neutron-drip density $\left(4.3 \times 10^{11} \mathrm{~g} \mathrm{~cm}^{-3}\right)$, neutrons emerge from the nuclei to form a free neutron superfluid. This density marks the boundary between the inuer and outer crust. The quantities that describe the neutron superfluid in the inner crust are calculated following the prescription given by Epstein (1988). The densities and pressures in and around the inner crust are shown in Fig. 1.

In Table 1, we present results for several low-order $l=2$ spheroidal modles including the effects of superfluidity, as well as results for those without superfluidity, where $\bar{\sigma} \equiv \sigma \sqrt{R_{*}^{3} / G M_{*}}$. The results are quite dramatic. The increased speed of the transverse waves, $c_{t}=\left(\mu / \rho_{N}\right)^{1 / 2}$, greatly changes the frequency spectrum of the shear modes trapped in the crust. It changes the frequency spectrum of acoustic modes as well. 
Table 37.1. The dimensionless frequency $\bar{\sigma}$ and the period $\Pi$ for surface $g$-modes, $i$-modes, $s$-modes and $p$-modes for $l=2$.

\begin{tabular}{lcccc}
\hline Mode & $\bar{\sigma}_{1}$ & $\Pi_{1}$ & $\bar{\sigma}_{2}$ & $\Pi_{2}$ \\
\hline$g_{2}$ & $2.037(-3)$ & 368.43 & $2.037(-3)$ & 368.43 \\
$g_{1}$ & $3.230(-3)$ & 232.38 & $3.230(-3)$ & 232.38 \\
\hline$i_{2}$ & $8.282(-3)$ & 90.626 & $8.282(-3)$ & 90.626 \\
$i_{1}$ & $1.028(-1)$ & 7.2956 & $1.716(-1)$ & 4.3738 \\
\hline$s_{1}$ & $3.093(-1)$ & 2.4266 & $7.015(-1)$ & 1.0700 \\
$s_{2}$ & $5.556(-1)$ & 1.3509 & $1.183(+0)$ & 0.6344 \\
$s_{3}$ & $7.483(-1)$ & 1.0030 & $1.437(+0)$ & 0.5224 \\
$s_{4}$ & $8.571(-1)$ & 0.8740 & $1.728(+0)$ & 0.4344 \\
\hline$F$ & - & - & $2.065(+0)$ & 0.3635 \\
$f$ & $1.886(+0)$ & 0.3979 & $1.482(+0)$ & 0.5064 \\
$p_{1}$ & $4.038(+0)$ & 0.1859 & $4.893(+0)$ & 0.1534 \\
$p_{2}$ & $4.736(+0)$ & 0.1585 & $6.231(+0)$ & 0.1205 \\
$p_{3}$ & $6.062(+0)$ & 0.1238 & $7.837(+0)$ & 0.0958 \\
$p_{4}$ & $7.129(+0)$ & 0.1053 & $9.755(+0)$ & 0.0768 \\
\hline \hline
\end{tabular}

The quantities $\bar{\sigma}_{1}$ and $\Pi_{1}$ are without superfluidity, while $\bar{\sigma}_{2}$ and $\Pi_{2}$ are with superfluidity in the inner crust. The periods $I$ are given in $\mathrm{ms}$.

Fig. 2 shows the radial component of the displacement vector, $\xi_{r} / r$, for $f$-modes (modes with no nodes of $\xi_{r} / r$ ) with and without superfluidity in the inner crust. We have two $f$-modes in the case with superfluidity. One of the two $f$-modes, denoted by $F$ in Table 1, has a large amplitude $\xi_{r} / r$ for the superfluid component, which is comparable with that of the lattice. Note that the oscillation amplitudes of the superfluid component are usually quite small compared with those of the lattice. In particular, this is true for the conventional $f$-mode, denoted by $f$ in Table 1 .

As shown by Table 1 , the surface $g$ modes are not affected at all by superfluidity in the inner crust.

\subsection{Conclusions}

We have computed several low-order, global $l=2$ spheroidal oscillation modes of neutron stars with $M=0.503 M_{\odot}$, including the effects of superfluidity. The presence of superfluid neutrons in the inner crust increases the local transverse wave speed substantially. This decreases the global oscillation periods of those modes which have significant amplitudes in the 

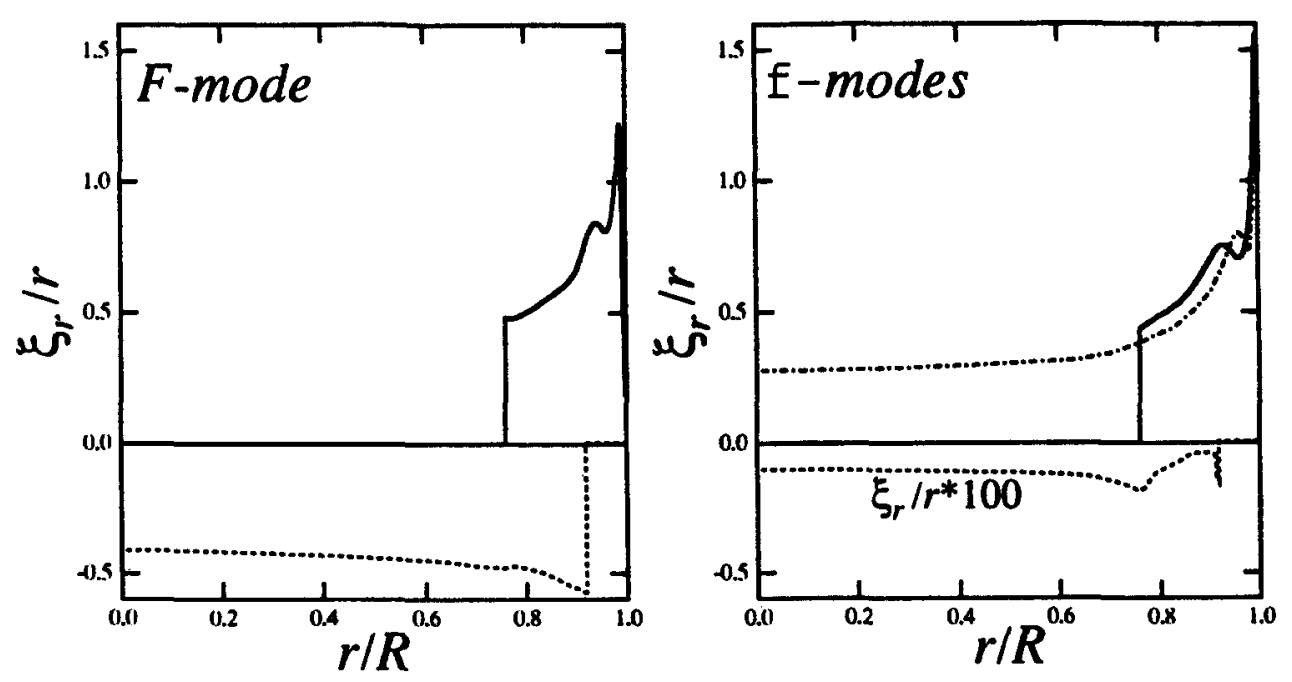

Fig. 37.2 The eigenfunctions $\xi_{r} / r$ of the $F$ - and $f$-modes. In each panel, the solid and dotted lines denote the eigenfunctions associated with the normal mat.ter and the superfluid, respectively. In the right-hand panel, the dash-dotted line is the $f$-mode for the case without superfiuidity in the inner crust.

inner crust, often by very large factors, thus effectively restricting motions associated with the pulsations to the outer crust. Conversely, modes which do not have significant amplitudes in the inner crust, such as the surface y-modes, are completely unaffected.

\section{Acknowledgements}

This work has been supported in part by the National Science Foundation under grant AST 91-15132, in part by NASA grant NAGW-2444, both through the University of Rochester, and in part by the U.S. Department. of Energy.

\section{References}

Baym, G., and Pines, D., Annuls of Phys., 66, 816, (1971)

Epstein, R. I., Ap. J., 333, 880, (1988)

Mc.Dermott, P. N., Van Horn, H. M., and Hansen, C. J., Ap. J., 325, 725, (1988)

Mendell, G., Ap. J., 380, 515, (1991)

Negele, J. W., and Vautherin, D., Nucl. Phys., A207, 298, (1973)

Vau Horn, H. M., and Epstein, R. I., Bull. A. A. S., 22, 748, (1990) 